\title{
DESPUÉS DE LA LEY 11/2007. ARCHIVOS Y ARCHIVEROS EN LA ADMINISTRACIÓN ELECTRÓNICA
}

\author{
Julio Cerdá Díaz* \\ Servicio de Archivos y Gestión Documental. Ayuntamiento de Arganda del Rey (Madrid).
}

Resumen: La Ley 11/2007, de 22 de junio, de acceso electrónico de los ciudadanos a los Servicios Públicos, obliga a las entidades públicas a la creación de canales de comunicación no presenciales entre la administración y los ciudadanos. Es el punto de partida de lo que conocemos como e-administración, un cambio de modelo en los entornos de trabajo que ha obligado a la redefinición de los conceptos, técnicas y procesos relacionados con la gestión documental. Ha sido en la administración local, la más próxima a los ciudadanos, donde los programas de modernización se han hecho más evidentes, siempre con dos objetivos clave: eficacia y transparencia, y con un núcleo central, las políticas de gestión de documentos electrónicos que afectan de lleno a los servicios de archivo.

Palabras clave: administración electrónica; documento electrónico; gestión electrónica; archivo electrónico; gestión documental; administración local; transparencia; gobierno abierto.

\section{Title: AFTER LAW 11/2007. ARCHIVES AND ARCHIVIST IN} EGOVERNMENT.

Abstract: Law 11/2007, of June 22nd, of electronic access of the citizens to the Public Services regulates the creation OF communication channelS between the administration and the citizens. This is the starting point for an e-Government project. In this article we explore the key paradigm shifts in a records management system implementation, that has forced the redefinition of the concepts, techniques and processes. Local governments should be able to use different and creative methods to run efficiently and economically and get the most modernization programs always with two key objectives: efficiency and transparency. The central core of their policies are the electronic records management.

Keywords: eGovernment; electronic document; electronic management; record management; local government; transparency; open government.

\section{INTRODUCCIÓN. BALANCE DE LOS PRIMEROS AÑOS DE E- ADMINISTRACIÓN.}

Han transcurrido cinco años desde la entrada en vigor de la Ley 11/2007, de 22 de junio, de acceso electrónico de los ciudadanos a los servicios públicos ${ }^{1}$, periodo suficiente para realizar balance de su nivel de aplicación en las administraciones locales. Si bien es cierto que la gestión electrónica de documentos ya era un tema recurrente desde mucho

*julcerda@gmail.com

Recibido: 22/10/2012; 2a versión: 12/12/2012; aceptado: 13/12/2012

CERDÁ DÍAZ, J. Después de la Ley 11/2007. Archivos y archiveros en la administración electrónica. Anales de Documentación, 2013, vol. 16, nº 1.

Disponible en: http://dx.doi.org/10.6018/analesdoc.16.1.161271 
tiempo atrás ${ }^{2}$, ha sido en los últimos años cuando se ha convertido en una prioridad para los ayuntamientos españoles. El nuevo marco legal ha dado lugar a incontables iniciativas estatales, autonómicas y locales que han intentado promover un cambio completo del modelo de relaciones entre la administración y los ciudadanos. En su título primero, la Ley 11/2007 configura los derechos de los ciudadanos en su relación electrónica con la administración, preservando siempre las garantías jurídicas reconocidas en la Ley 30/1992. Entre esos derechos destacan los siguientes:

- Derecho a elegir, entre aquellos que en cada momento se encuentren disponibles, el canal para relacionarse por medios electrónicos con las administraciones

- Derecho a no aportar los datos y documentos que obren en poder de las administraciones públicas, y que lleva implícito la imprescindible interoperabilidad entre las administraciones públicas ${ }^{3}$

- Derecho a la igualdad en el acceso electrónico a los servicios de las administraciones públicas

- Derecho a conocer por medios electrónicos el estado de tramitación de los procedimientos. Directamente relacionado con el desarrollo de plataformas de tramitación de expedientes, sedes electrónicas y portales del ciudadano

- Derecho a obtener copias electrónicas de los documentos electrónicos

- Derecho a la conservación en formato electrónico de los documentos electrónicos que formen parte de un expediente. Es el derecho que más directamente nos afecta. Nos exige la difícil tarea de buscar los medios para garantizar la accesibilidad y conservación permanente de los datos y metadatos que forman parte de cada procedimiento

- Derecho a utilizar los medios y sistemas de identificación electrónica

- Derecho a la seguridad y confidencialidad de los datos. Las anheladas interoperabilidad e intraoperabilidad, el compartir datos y registros entre las administraciones públicas o las diversas áreas municipales, tienen que ir unidas a un aumento de los controles para proteger la pérdida, alteración o acceso no autorizado de los datos de carácter personal.

Un conjunto de derechos que, consecuentemente, deberían dar lugar a un intenso programa de actuaciones. Se fijaba una fecha para hacer efectivo su cumplimiento, según refleja su disposición final tercera: el 31 de diciembre de 2009. El año 2010 era el momento indicado para iniciar una nueva era en la administración pública española, aunque se dejaba una puerta abierta para su no aplicación: "siempre que lo permitan las disponibilidades presupuestarias" ". Si se ha utilizado este pretexto en el bienio 20072009, cuando los presupuestos municipales y las inversiones públicas vivieron su momento más expansivo, resulta difícil pensar que puedan cambiar las dinámicas en el actual escenario de recesión y recortes presupuestarios, aunque el condicionante económico se debería situar en su justo lugar y no convertirse en una coartada para la inacción ${ }^{5}$. Es cierto que cuando teníamos mucho no hicimos demasiado, y ahora que tenemos poco es cuando se nos exigen resultados, por eso ahora, más que nunca, son tan 
importantes la proyectos en colaboración, el compartir recursos entre administraciones, o priorizar las estrategias de desarrollo a partir de software no propietario con herramientas de fuentes abiertas.

En cualquier caso, a nadie se le escapa una realidad incontestable. ¿Son las administraciones locales que han contado con más medios económicos, aquellas que han adjudicado contratos de mayor importe, las que hoy cuentan con las mejores y más eficientes soluciones de gestión electrónica? No siempre es así. El principal factor de éxito son los equipos humanos que impulsan cada uno de los proyectos. Más importante que la tecnología o los recursos, será la implicación de toda la organización en ese objetivo común, comenzando por el imprescindible liderazgo político que respalde un plan director asumido por todas las áreas municipales. Son necesarios procesos de tanto calado que no se pueden poner en marcha simplemente con la entrada en vigor de una norma legal o la adquisición de un determinado sistema de gestión electrónica. Las nuevas plataformas implican cambios de hábitos adquiridos durante décadas de trabajo aislado de las distintas áreas administrativas, sin compartir ni depender, y si algo caracteriza a los nuevos sistemas es el trabajo colaborativo y la dependencia tecnológica, aparecen nuevas funciones y se exigen también nuevas habilidades.

El primer paso es conocer en qué lugar nos encontramos. A nivel europeo se ha conseguido un notable avance en los últimos años. Así lo reflejan los estudios presentados en la VI Conferencia Ministerial de eGoverment ${ }^{6}$ donde se tomó como referencia la declaración de la conferencia anterior celebrada en el 2009 en Malmö (Suecia) ${ }^{7}$. Según las conclusiones presentadas, el $71 \%$ de los servicios públicos europeos son accesibles por medios electrónicos y el $85 \%$ de sus ciudadanos acceden a ellos habitualmente ${ }^{8}$. España ocuparía la posición 13 del ranking europeo de eGoverment, y según otros análisis ${ }^{9}$, el 23 a escala mundial.

Centrándonos en la situación de la administración local, los datos del informe eEspaña 2011 elaborado por la Fundación Orange nos ofrece la disponibilidad de los servicios públicos online en los veinte mayores ayuntamientos españoles ${ }^{10}$. El porcentaje de disponibilidad media de los once servicios públicos objeto de estudio es del $72 \%$. Cabe destacar que los servicios asociados a fines recaudatorios (pago de impuestos y multas) son los más desarrollados, por el propio interés económico de la administración y porque no suele ser necesario el uso de certificado electrónico o DNIe por parte del ciudadano. La escasa utilización del DNI electrónico es quizás lo más preocupante del estudio y debería ser motivo de profunda reflexión. Del $27 \%$ de la población española que ya tiene en sus manos un DNIe, tan solo el $4,7 \%$ de los usuarios lo utilizan para realizar de forma telemática trámites administrativos. ¿Se utiliza poco el DNIe por un problema de hábitos culturales o por la reducida oferta de servicios electrónicos de calidad?

El paisaje del entorno electrónico se vuelve mucho más desolador cuando hablamos de pequeños y medianos municipios, el grueso de los ayuntamientos españoles. El grado de adaptación de las entidades locales de menor población a la administración electrónica es 
"bajo o muy bajo". Es la expresión que utiliza un estudio impulsado por la Federación Española de Municipios y Provincias (FEMP) que ha analizado el impacto de la Ley $11 / 2007$ en los ayuntamientos de menos de 20.000 habitantes $^{11}$. Un panorama que concede aún más valor a los proyectos de modernización con un alto nivel de excelencia que han impulsado algunos de estos ayuntamientos, y que deberían ser un referente para los que aún están en las primeras fases de sus procesos de cambio ${ }^{12}$.

Lo cierto es que menos del $25 \%$ de estos municipios han aprobado ordenanzas de administración electrónica, y el $80 \%$ de los procedimientos administrativos no tienen desarrollada la tramitación electrónica, siendo nuevamente los relacionados con servicios tributarios los que están más implantados, junto a los servicios demográficos, territoriales y urbanísticos. Destaca por su importancia la escasa implantación del procedimiento de contratación electrónica, quizás motivado por la complejidad de su trámite, a pesar de que el art. 42 de la Ley de Contratos del Sector Público obligaba a que estuviera en pleno funcionamiento a partir del 1 de mayo de $2008^{13}$.

La conclusión de todos los estudios parece muy clara, los resultados confirman lo que era una impresión general: no se ha cumplido la hoja de ruta que establecía la Ley 11/2007. Han pasado cinco años y aún estamos a escasos metros de la línea de partida. La diversidad es la principal característica de los ayuntamientos españoles, era fácil de prever que la plena disponibilidad de servicios electrónicos iba a llevar mucho más tiempo que lo que establecían las previsiones iniciales. Los cambios culturales se producen mucho más despacio que los tecnológicos. En cualquier caso, acercar más la administración a los ciudadanos y lograr, en última instancia, la interoperabilidad entre las administraciones públicas, es sin duda un nuevo e ilusionante reto. Quizás no nos deba importar tanto los resultados obtenidos, como lo mucho que se puede llegar a conseguir. Es más importante identificar correctamente el horizonte que caer en el desánimo por los problemas del presente, aprender a solucionarlos es parte del trayecto.

\section{EL SERVICIO DE ARCHIVO EN LAS PLATAFORMAS DE TRAMITACIÓN ELECTRÓNICA.}

Antes de intentar definir nuestro territorio de actuación no está de más reflexionar sobre las funciones del archivo. Es un tema que hasta hace unos años apenas generaba debate, pero ahora, en los albores de la era digital, en la edad de oro de la gestión de la información, aparecen nuevas tareas técnicas, nuevos espacios de trabajo y por tanto nuevos matices en los trazos que definen la profesión. ¿Todos tenemos los mismos objetivos? ¿Cuál es la prioridad? ¿Qué es lo más importante? ¿Cuál es la función del archivo?

Nadie duda del lugar protagonista que siempre ha ocupado el archivo en la gestión administrativa. En el Corpus Iuris Civilis del Emperador Justiniano redactado en la primera mitad del siglo VI, es la base del derecho europeo, se recoge una interpretación del valor de los archivos que se puede calificar de intemporal: "quatenus incorrupta 
maneant et velociter inveniantur a requirentibus", "donde los documentos permanezcan incorruptos y con rapidez sean encontrados cuando se precisen"14. Una definición que recoge los valores esenciales de un archivo: garantizar la conservación permanente y el acceso rápido, ágil y pertinente a los documentos de su organización. Que en el siglo VI se introduzca el término "velociter" lo dice todo, el jurista, la administración, ya exigía lo que ahora es un eje estratégico de los planes de modernización, aumentar la eficacia, prestar más y mejor servicio, del modo más eficiente. En quince siglos no cambiaron mucho las cosas, era suficiente con conocer las técnicas de archivo o los principios de conservación del pergamino o del papel, y asunto concluido. Los documentos llegaban puntualmente a sus usuarios cuando nos los solicitaban.

Ahora tenemos la enorme suerte de estar viviendo un cambio de época, no es sólo una época de cambios, pasarán varias generaciones antes que se produzca una revolución similar. Cuando en una disciplina se produce una fase de transición como la que estamos viviendo, y que al menos durará un par de décadas más, es propio de la naturaleza humana que aparezcan dos tipos de actitudes a la hora de afrontar los nuevos retos:

- Tendencia conservadora o reactiva. Aparece el instinto de conservación. Existe una atadura emocional con el concepto tradicional de archivo. Es una reacción natural por lo que es la postura más frecuente. Surge el temor de que otros ocupen nuestro lugar, nos sentimos vulnerables o incluso prescindibles, y aparecen conductas defensivas o de cautela frente al riesgo que implica lo nuevo, todo son dificultades o amenazas. Un planteamiento que lleva a ver sólo los problemas de cualquier proyecto de innovación, y se habla más de pasado, y con formas del pasado, que de futuro.

- Tendencia expansiva o proactiva. Prevalece el instinto de exploración, de disfrute de la novedad y el riesgo, la curiosidad que conduce a la creatividad. No se ponen fronteras al archivo y se aprovecha la oportunidad que ofrecen los sistemas de gestión electrónica para aprender y luego emprender. El objetivo, el santo grial, junto con la conservación, es esa "velociter" en la gestión de documentos que indicaba en el siglo VI el Código de Justiniano. El objetivo es aprender de los inevitables errores, saber convivir con las dificultades, trabajar en equipos muy heterogéneos donde lo más importante será lo que se puede aportar, y traducirlo en acciones concretas y visibles, y sobre todo, siempre bajo la bandera de la perseverancia, tozudamente positivos.

Son dos opciones igual de respetables. Las dos posturas tienen que coexistir, son necesarias por una pura cuestión de equilibrio. Si bien habrá que valorar que el área de centralidad de la gestión documental está en las plataformas de gestión electrónica y que lo servicios de archivo convencionales serán desplazados a zonas más periféricas del organigrama. Lo más inteligente es atemperar las actitudes defensivas o poco flexibles 
ante las corrientes de cambio porque pueden producir aislamiento, y a medio plazo, una paulatina pérdida de visibilidad y capacidad de influencia en la organización.

Pensando en archivos de la administración local, los más próximos y relacionados con la gestión administrativa, y salvo aquellos que custodien un muy notable patrimonio documental, son servicios que están llamados a transformarse completamente. Tendrán que evolucionar más en los próximos años de lo que lo han hecho en el último milenio, se puede discrepar sobre el plazo de ese proceso, pero nadie puede dudar que la cuenta atrás ya ha comenzado ${ }^{15}$.

\section{EL NUEVO CONTEXTO DE TRABAJO. GESTIÓN DE DOCUMENTOS Y ARCHIVOS ELECTRÓNICOS.}

No podemos diseñar una hoja de ruta sin identificar correctamente el destino, sin definir bien los objetivos, y la prioridad es adaptarlos al tipo de servicios que esperan recibir los ciudadanos. Recientemente se ha aprobado una nueva agenda digital para España, un marco de referencia abierto a propuestas, que pretende impulsar un uso eficaz e intensivo de las tecnologías y aprovechar las oportunidades generadas por una economía cada vez más digitalizada y centrada en la Red. Su punto tercero está dedicado al desarrollo de la administración electrónica, y establece con claridad las prioridades: "incrementar la productividad de nuestras administraciones para conseguir una reducción del gasto público, manteniendo al mismo tiempo unos servicios públicos universales y de calidad", y para ello se proponen tres medidas muy concretas ${ }^{16}$ :

- Simplificar y eliminar las cargas administrativas innecesarias, realizando los cambios normativos precisos, reduciendo trámites y haciendo efectivo el derecho de ciudadanos y empresas de no aportar datos que estén en poder de la administración.

- Desarrollar una estrategia de gobierno abierto en nuestras administraciones públicas que potencie la creación de valor público mediante:

- Transparencia, dando acceso a la información y resultados obtenidos en la actividad administrativa ${ }^{17}$

- Participación ciudadana en las decisiones de la administración, permitiendo recoger sugerencias, comentarios y críticas

- Colaboración con ciudadanos, empresas y administraciones en el diseño, implementación y prestación de servicios digitales.

- Elaborar una estrategia y un plan de acción de reutilización de la información del sector público para permitir el desarrollo de servicios de alto valor que contribuyan al impulso de la actividad económica y/o la generación de servicios de valor para ciudadanos y empresas ${ }^{18}$. 
Unas medidas que deben ser el eje rector de los programas de modernización y que se pueden resumir en dos conceptos clave: eficacia y transparencia. En todos los casos el núcleo central son las políticas de gestión de documentos electrónicos, y afectan por tanto de lleno a los servicios de archivo. A modo de aproximación, teniendo en cuenta que las plataformas de tramitación integran muy diversas capas, vamos a intentar concretar las medidas o ámbitos de actuación donde nuestro trabajo puede ser más relevante.

Es relativamente sencillo responder a la pregunta ¿qué hacer?, pero entramos en un territorio bien distinto, el de la incertidumbre, cuando pretendemos responder a la pregunta que realmente nos interesa ¿cómo hacerlo? En el ámbito de la gestión documental las fórmulas magistrales no existen, son tantas las singularidades de cada proyecto, los distintos elementos que puedan entrar en juego, que no podemos pretender buscar soluciones antes de que se produzcan. Los contenidos y procesos recogidos en la Norma Técnica de Interoperabilidad de Política de gestión de documentos electrónicos ${ }^{19}$ nos van a servir de guía.

Las normas técnicas publicadas recogen un conjunto de directrices sobre digitalización, expediente electrónico, copiado auténtico y conversión, política de firma, estándares, intermediación de datos, modelos de datos, gestión de documentos electrónicos, o el modelo de datos para el intercambio de asientos registrales. Son los elementos prácticos y operativos que pretenden asegurar la interoperabilidad con el ciudadano y entre las administraciones públicas. Por si hubiera alguna duda, en su punto cuarto, actores involucrados en la aplicación de la norma, no se hace mención expresa al término "archivero" pero sí a la funciones que secularmente hemos desempeñado: "gestión y conservación documental": "El personal responsable de la planificación, implantación y administración del programa de tratamiento de documentos y sus operaciones, cualificado, dedicado e instruido en gestión y conservación documental y que participará en el diseño, implementación y actualización de los sistemas de gestión y conservación documental". En el punto quinto se concreta además que el programa "se aplicará de manera continua sobre todas las etapas o periodos del ciclo de vida de los documentos y expedientes electrónicos para los que garantizará su autenticidad, integridad, confidencialidad, disponibilidad y trazabilidad; permitiendo la protección, recuperación y conservación fisica y lógica de los documentos y su contexto".

Una vez más se vuelven a recordar los requisitos técnicos que deben cumplir los documentos electrónicos, tal como se establece en el art. 1 de La Ley 11/2007, asegurando la disponibilidad, el acceso, la integridad, la autenticidad, la confidencialidad y la conservación de los datos. No está de más, toda solución de gestión electrónica debería pasar una auditoría, antes de ser implementada que pudiera garantizar que cumplen con esos requerimientos, y que en caso de ser necesario pudiera demostrarlo y certificarlo ante organismos superiores o tribunales. Su incumplimiento puede suponer un perjuicio muy grave para la administración, por la imposibilidad de atender alguna de sus obligaciones fundamentales, perjudicando los derechos de los ciudadanos, o afectando gravemente a la gestión y a la memoria corporativa de la administración. 
Una posible solución sería que las administraciones recurrieran a una entidad certificadora y auditasen su sistema de gestión de documentos electrónicos en las distintas normas relacionadas con la gestión de documentos y seguridad de la información, y en particular en la serie de normas ISO 30300 donde las buenas prácticas de la ISO anteriores se convierten en requerimientos ${ }^{20}$. La exigencia de cumplir con los requisitos de las normas se debería trasladar igualmente a las herramientas de gestión de los numerosos proveedores que ofertan soluciones de gestión electrónica ${ }^{21}$. No son pocos los ayuntamientos que han confiado todas sus herramientas de gestión a alguna de las empresas del sector, en ocasiones sin las suficientes garantías, y sin que finalmente hayan obtenido los resultados esperados. En el caso de una eventual pérdida de datos, de los documentos que testimonian una determinada actuación administrativa, ¿quién sería el responsable? ¿la empresa contratada que ha desarrollado esa plataforma de gestión electrónica o la administración contratante que tomó la decisión de utilizarla?

\subsection{Soluciones de archivo electrónico. El módulo de conservación.}

Con el único objetivo de ofrecer unas orientaciones generales vamos a recordar los requisitos técnicos que deben tener los documentos y repositorios electrónicos, y los problemas más habituales que nos solemos encontrar a la hora de su gestión (Tabla I):

\begin{tabular}{|l|l|l|}
\hline \multicolumn{1}{|c|}{$\begin{array}{l}\text { Requisitos técnicos } \\
\text { Ley } 11 / 2007\end{array}$} & $\begin{array}{l}\text { Problemas más frecuentes } \\
\text { Archivos y repositorios electrónicos }\end{array}$ \\
\hline $\begin{array}{l}\text { Disponibilidad "Teniendo en } \\
\text { cuenta la obsolescencia tanto de } \\
\text { los sistemas informáticos como los } \\
\text { soportes electrónicos una de las } \\
\text { características esenciales de un } \\
\text { repositorio es su disponibilidad al } \\
\text { margen del sistema de gestión } \\
\text { documental o de las tecnologías de } \\
\text { creación". }\end{array}$ & $\begin{array}{l}\text { La localización y acceso a los documentos sólo } \\
\text { se puede realizar a través del framework del } \\
\text { procesos de búsqueda o migración de datos y } \\
\text { metadatos al margen del sistema propietario. El } \\
\text { objetivo debe ser la total independencia respecto } \\
\text { a tecnologías y proveedores concretos. } \\
\text { La estructura o "file system" del repositorio es } \\
\text { aleatoria. No es posible comprender la lógica de } \\
\text { creación de los directorios } \\
\text { Los formatos de los documentos electrónicos no } \\
\text { garantizan su conservación a largo plazo. No } \\
\text { están previstos procesos de cambios de formato } \\
\text { para evitar la obsolescencia y la no dependencia } \\
\text { de las tecnologías de creación. } \\
\text { No existe un protocolo o plan para la } \\
\text { conservación de documentos. Todos los } \\
\text { documentos se tratan por igual, no existe una } \\
\text { política de conservación, migración, conversión } \\
\text { o de identificación de los documentos } \\
\text { esenciales. }\end{array}$ \\
\hline
\end{tabular}




\begin{tabular}{|c|c|}
\hline & $\begin{array}{l}\text { - La nomenclatura de los documentos electrónicos } \\
\text { es modificada de modo aleatorio en el momento } \\
\text { de llegar al repositorio. }\end{array}$ \\
\hline $\begin{array}{l}\text { Autenticidad "Con la } \\
\text { autenticación de los documentos se } \\
\text { acredita la identidad de las } \\
\text { personas o entidades que han } \\
\text { participado en las operaciones, } \\
\text { transacciones y actos } \\
\text { administrativos, garantizando su } \\
\text { autoría e integridad". }\end{array}$ & $\begin{array}{l}\text { - Caducidad de los certificados electrónicos. No } \\
\text { existen garantías de la validez de las firmas y } \\
\text { certificados digitales en el transcurso del tiempo. }\end{array}$ \\
\hline $\begin{array}{l}\text { Integridad "Capacidad } \\
\text { mantener los datos y los } \\
\text { documentos } \\
\text { modificaciones no autorizadas. } \\
\text { Una de las vías para garantizar la } \\
\text { integridad del procedimiento } \\
\text { electrónico es la inclusión de una } \\
\text { relación de documentos, en forma } \\
\text { de indice electrónico, donde se } \\
\text { relacionarán los documentos que } \\
\text { forman parte de ese proceso, } \\
\text { indicando el estado de tramitación, } \\
\text { documentos y actos administrativos } \\
\text { que lo componen". }\end{array}$ & $\begin{array}{l}\text { - El repositorio electrónico no responde a lo que } \\
\text { exige la ley, ya que no incorpora los metadatos } \\
\text { de los documentos o índices del contenido de los } \\
\text { procedimientos. Para ambos casos, la solución } \\
\text { más habitual es la inclusión de ficheros XMP y } \\
\text { XML. }\end{array}$ \\
\hline $\begin{array}{l}\text { Confidencialidad "La } \\
\text { confidencialidad es la propiedad de } \\
\text { un sistema para prevenir el acceso } \\
\text { de información a personas o } \\
\text { sistemas no autorizados". }\end{array}$ & $\begin{array}{l}\text { - No se definen los controles de acceso a los } \\
\text { diferentes tipos de información en función de su } \\
\text { nivel de sensibilidad según la normativa de } \\
\text { protección de datos. }\end{array}$ \\
\hline $\begin{array}{l}\text { Trazabilidad "Capacidad de } \\
\text { conocer y reproducir a través de } \\
\text { medios electrónicos, la información } \\
\text { relativa al estado de tramitación y } \\
\text { actos administrativos, tanto de los } \\
\text { documentos como de sus } \\
\text { metadatos, que forman parte de un } \\
\text { determinado procedimiento". }\end{array}$ & $\begin{array}{l}\text { - Relacionado con la ausencia de los índices y los } \\
\text { metadatos de los documentos en el repositorio. } \\
\text { Hace imposible acceder a los actos } \\
\text { administrativos o trámites relacionados al } \\
\text { margen de las herramientas propias del gestor } \\
\text { documental. }\end{array}$ \\
\hline
\end{tabular}

Tabla I. Problemas más frecuentes en la gestión de archivos electrónicos. 
Muchas de estas cuestiones vienen originadas por la inexistencia de un modelo de archivo electrónico. Es una práctica habitual que se desarrollen gestores documentales, las plataformas que soportan la tramitación electrónica, los servicios más visibles para el ciudadano, y se deje para una fase o proyecto posterior el imprescindible módulo de archivo electrónico para la conservación permanente de documentos. En la mayoría de las soluciones de gestión implantadas se ha relacionado el término archivo con "sistema de almacenamiento". Poco más que un "file system" gestionado desde el "framework" o capa de desarrollo del gestor, sin abordar el desarrollo de procesos o herramientas que garanticen la plena disponibilidad de los datos y metadatos, al margen de cualquier sistema, y su conservación a largo plazo.

Es necesario recordar lo que se entiende por "archivo electrónico", lo define la propia norma: "el repositorio electrónico, complementario y equivalente en cuanto a su función a los archivos convencionales, destinado a cubrir el conjunto del ciclo de vida de los documentos electrónicos, y donde se administrarán datos y documentos electrónicos, y sus metadatos "22. La obsolescencia tecnológica es un hecho inevitable, y un módulo de archivo está específicamente diseñado para que, en el peor de los escenarios posibles, se pueda garantizar la accesibilidad, disponibilidad, integridad y autenticidad de los documentos electrónicos, independientemente del medio físico de almacenamiento, la tecnología empleada para su creación o el formato del fichero.

El diseño de ese módulo de archivo electrónico estará condicionado por el volumen de información gestionada, pero con carácter general, y para facilitar las tareas de control, selección y evaluación, deberá existir un repositorio de gestión, asimilable a los antiguos "archivos de gestión o de oficina" donde se almacenan los datos y metadatos de los documentos en trámite o resueltos, y por otro lado, un repositorio de archivo definitivo. Ambos repositorios deberían ser accesibles desde el sistema de recuperación del gestor documental. La transferencia de los datos y metadatos de un repositorio a otro implicaría también el traspaso de responsabilidades sobre su conservación y preservación, aunque las políticas de conservación se aplicarían desde el mismo instante en que se genera el documento, no sólo en el momento de su traspaso al archivo electrónico definitivo ${ }^{23}$.

Una confusión habitual consiste en no diferenciar entre un Sistema de Gestión de Documentos, la labor que hacen los gestores documentales que se han desarrollado en los últimos años, de los Sistemas de Gestión de Documentos Electrónicos de Archivo que, dada su relevancia y las funcionalidades técnicas que incorporan, tiene que ser un módulo independiente. Se suelen dar por suficientes los sistemas de almacenamiento de los gestores documentales, cuando tienen como único objetivo servir de repositorio de trabajo para las múltiples tareas que requieren las plataformas de tramitación electrónica. Cumplen con una función que es imprescindible en los procesos de tramitación electrónica, la de almacenar los numerosos datos y metadatos que se van generando en los procesos de tramitación electrónica, y por ello incluyen borradores, distintas versiones de los documentos, duplicados sin valor jurídico, o documentos generados electrónicamente, pero que en ningún caso deben ser conservados una vez resuelto el procedimiento. 
Una realidad muy distinta es un módulo de conservación de documentos que sólo admite documentos finalizados y de conservación permanente, donde se encapsulan los ficheros electrónicos junto con sus metadatos, índices y signos de validación en un paquete e identificador único. Son unos "paquetes de información" que pasarán a ser las unidades de almacenamiento del sistema de archivo $^{24}$. En la actualidad, el modelo de referencia de archivo electrónico para la preservación a largo plazo es OAIS (Open Archive Information System) e incluye los siguientes módulos funcionales: ingreso (ingest), almacenamiento (storage), gestión de datos (data management), consulta y recuperación (access), administración (administration) y preservación (preservation planning $)^{25}$. Además debería contar con otras utilidades como validaciones, extracción de metadatos, generación de representaciones de los contenidos, o la posibilidad de exportar los resultados de las búsquedas en distintos formatos de ficheros estructurados y reutilizables. Si un módulo de esas características, o con unas funciones similares, no existe en la plataforma de gestión, podemos decir que, en los términos establecidos por ley, no existe archivo electrónico, y por las responsabilidades que en el futuro pudieran derivarse, la situación debería ser puesta en conocimiento del órgano de gobierno competente.

En cierto modo, podríamos estar trasladando al entorno electrónico aquella situación que siempre aparecía en los manuales tradicionales de archivística cuando nos describían la diferencia entre un "almacén de papel" y un "archivo", en los dos espacios se conservaban documentos pero sólo uno se puede considerar un archivo. Si no existe un módulo de archivo en la plataforma de gestión electrónica no puede haber archivero ¿quién es entonces el responsable técnico de la conservación de esos documentos?

\subsection{Gestión de documentos electrónicos. Procesos técnicos.}

Participar y colaborar en las plataformas de tramitación electrónica exige algunas adaptaciones de los métodos habituales de trabajo, uno de los cambios más relevantes tiene que ver con la unidad de gestión. En los servicios de archivo la unidad de gestión más utilizada es la unidad documental, simple y compuesta, y por tanto las tareas de identificación, valoración, clasificación y descripción se hacen normalmente con expedientes o con series documentales. Sin embargo, en las plataformas de tramitación electrónica sólo se trabaja con lo que nosotros llamamos unidades documentales simples, el expediente no es más que una unidad virtual, y en el gestor se representa por un índice que referencia los documentos electrónicos que lo componen, así lo indica la propia Ley $11 / 2007$. Con otro importante añadido, un mismo documento electrónico puede formar parte de varios índices o expedientes electrónicos, una situación frecuente cuando se hace un uso correcto y eficaz de la plataforma de gestión.

¿Qué consecuencias tiene?, ninguna que no se pueda solucionar, nos obliga a crear mecanismos de relación entre expedientes y a un aumento de los controles en las posibles propuestas de selección y evaluación. Las tablas de valoración que conocemos están pensadas para series documentales o procedimientos concretos, ahora el punto de vista es 
otro y las propuestas de destrucción tendrán que ser mucho más meditadas. Ante el peligro de eliminar documentos utilizados en otros procesos habrá que identificar previamente los datos y metadatos que potencialmente puedan ser reutilizados ${ }^{26}$.

Otro problema es cuando intentamos poner en relación o compartir las bases de datos o las digitalizaciones realizadas desde el servicio de archivo con la plataforma de tramitación electrónica. Si una de las muchas virtudes del gestor es que posibilita que las áreas y departamentos municipales accedan fácilmente a sus procedimientos, estén en fase de trámite o hayan sido resueltos, lo natural es que desde la misma plataforma de consulta se pueda acceder a toda la información existente, haya sido tramitada por el gestor o sea de la era pre-electrónica y se conserve en el archivo. En ese momento nos encontramos de nuevo con el problema de la distinta unidad de gestión. El trabajo principal en los archivos ha sido describir y digitalizar expedientes, procedimientos completos, mientras que en los gestores documentales la unidad natural de gestión y recuperación son documentos únicos, aunque naturalmente esté presente la posibilidad de recuperar el índice completo de cada expediente.

El mismo caso nos encontramos cuando, a través de una sede electrónica, se ofrece a los ciudadanos y empresas el acceso a los documentos donde son parte interesada. No tiene ningún sentido que con su certificado digital sólo puedan acceder a los tramitados en plataforma, deberían poder acceder a todos los conservados por el ayuntamiento. ¿La solución?, si queremos compartir tenemos que adaptar, tanto los procedimientos de digitalización como el sistema de descripción. El referente a seguir serán los nuevos esquemas de metadatos, y tendrán prioridad las series documentales de alto impacto, las que llevan aparejada la concesión de licencias o derechos a los ciudadanos.

Hechas estas consideraciones, vamos a comentar brevemente lo que más nos ocupa y preocupa, el lugar del archivo en las plataformas de administración electrónica. Seguiremos los procesos técnicos que se relacionan en la Norma Técnica de Interoperabilidad de Política de gestión de documentos electrónicos (Tabla II):

\begin{tabular}{|c|c|}
\hline $\begin{array}{l}\stackrel{\text { ¿Qué hacer? }}{\text { Normas }} \text { Técnicas } \\
\text { Interoperabilidad. }\end{array}$ & $\begin{array}{l}\text { ¿Cómo hacerlo? } \\
\text { Orientaciones y reflexiones. }\end{array}$ \\
\hline $\begin{array}{l}\text { Captura "que incluirá el tratamiento } \\
\text { de los metadatos mínimos obligatorios } \\
\text { definidos en la Norma Técnica de } \\
\text { Interoperabilidad de Documento } \\
\text { Electrónico". }\end{array}$ & $\begin{array}{l}\text { La función de Registro tiene un notable } \\
\text { protagonismo en las plataformas de gestión } \\
\text { electrónica, es quizás el módulo donde más se } \\
\text { está avanzando: Se interconectan registros }\end{array}$ \\
\hline $\begin{array}{l}\text { Registro legal “que, además del } \\
\text { tratamiento de } \\
\text { electrónicos recibidos, atenderá a la }\end{array}$ & $\begin{array}{l}\text { presenciales con registros electronicos y } \\
\text { telemáticos (tanto de sede electrónica como } \\
\text { asientos de otras entidades registrales, aplicando } \\
\text { la Norma Técnica de Interoperabilidad de }\end{array}$ \\
\hline
\end{tabular}

Anales de Documentación, 2013, vol. 16, nº 1 


\begin{tabular}{|c|c|}
\hline $\begin{array}{l}\text { posibilidad de digitalizar documentos } \\
\text { en soporte papel según lo establecido } \\
\text { en la Norma Técnica de } \\
\text { Interoperabilidad de Digitalización de } \\
\text { Documentos". }\end{array}$ & $\begin{array}{l}\text { Modelo de Datos). } \\
\text { Tareas técnicas que el archivo debe supervisar: } \\
\text { Proceso de conversión de los documentos } \\
\text { presentados originalmente en papel en } \\
\text { documentos electrónicos, aplicando la Norma } \\
\text { Técnica de Interoperabilidad de Digitalización y } \\
\text { de Documento Electrónico. } \\
\text { Todo documento deberá ser identificado y tener } \\
\text { asociada la información adicional que permita } \\
\text { su contextualización. En Registro se hace una } \\
\text { primera identificación o categorización de los } \\
\text { documentos, son sus primeros metadatos. Antes } \\
\text { de su distribución es necesario asignarles una } \\
\text { tipología documental a los documentos } \\
\text { aportados por los ciudadanos, identificarlos en } \\
\text { el catálogo de procedimientos, y el área de } \\
\text { gestión que tiene las competencias del trámite. }\end{array}$ \\
\hline $\begin{array}{l}\text { Clasificación "que incluirá los } \\
\text { criterios de formación de expedientes y } \\
\text { agrupaciones de documentos } \\
\text { electrónicos según la Norma Técnica } \\
\text { de Interoperabilidad de Expediente } \\
\text { Electrónico, asi como la clasificación } \\
\text { funcional de acuerdo con el cuadro de } \\
\text { clasificación de la organización”. }\end{array}$ & $\begin{array}{l}\text { Las Normas Técnicas de Interoperabilidad dan } \\
\text { por hecho que debe existir un "cuadro de } \\
\text { clasificación funcional de la organización". El } \\
\text { objetivo es codificar todos los procedimientos } \\
\text { que se van a tramitar electrónicamente. El } \\
\text { cuadro será el resultado de un necesario paso } \\
\text { previo, el de análisis y, en muchos casos, } \\
\text { rediseño de procedimientos. } \\
\text { Lo habitual es que el cuadro de clasificación del } \\
\text { gestor sea una adaptación y desarrollo del } \\
\text { Cuadro de Clasificación del Archivo. Tienen } \\
\text { que estar muy relacionados, pero } \\
\text { inevitablemente van a aparecer nuevos } \\
\text { procedimientos específicos que habrça que } \\
\text { desarrollar con nuevas series y con nuevos } \\
\text { niveles de clasificación. En el caso de poder } \\
\text { llegar a emplear un cuadro único, las series } \\
\text { "históricas", trámites que ya no se realizan, no } \\
\text { estarán visibles en el gestor documental. }\end{array}$ \\
\hline $\begin{array}{l}\text { Descripción "que atenderá a lo } \\
\text { establecido en el apartado VII de esta } \\
\text { norma así como a la posible redacción } \\
\text { de un esquema institucional de } \\
\text { metadatos". }\end{array}$ & $\begin{array}{l}\text { Los esquemas de metadatos son sin duda el } \\
\text { elemento clave en los sistemas de gestión de } \\
\text { documentos electrónicos }{ }^{27} \text {. Todos los } \\
\text { documentos tienen que estar identificados y } \\
\text { tener asociada la información adicional que }\end{array}$ \\
\hline
\end{tabular}




\begin{tabular}{|c|c|}
\hline $\begin{array}{l}\text { "Las organizaciones garantizarán } \\
\text { la disponibilidad e integridad de los } \\
\text { metadatos de sus documentos } \\
\text { electrónicos, manteniendo de manera } \\
\text { permanente las relaciones entre cada } \\
\text { documento y sus metadatos". }\end{array}$ & $\begin{array}{l}\text { permita su contextualización, tanto en el } \\
\text { transcurso del trámite, como una vez concluido } \\
\text { y durante todo el tiempo que deba ser } \\
\text { conservado. Esa "información sobre la } \\
\text { información", independiente del documento, y } \\
\text { que permite categorizarlo y contextualizarlo, se } \\
\text { conoce como metadato. } \\
\text { Dependiendo de su función informativa pueden } \\
\text { ser descriptivos (tipología, datos identificativos, } \\
\text { interesados, fecha, localización, procesos del } \\
\text { trámite, marco legal, seguridad, protección de } \\
\text { datos, etc.), técnicos (información sobre } \\
\text { soportes y ficheros) o de preservación (con la } \\
\text { finalidad de garantizar la integridad y } \\
\text { disponibilidad de los contenidos, como por } \\
\text { ejemplo la validez y vigencia de las firmas). }\end{array}$ \\
\hline $\begin{array}{l}\text { Acceso "que contemplará la posible } \\
\text { regulación institucional de dicha } \\
\text { práctica asi como la trazabilidad de las } \\
\text { acciones que se realizan sobre cada } \\
\text { uno de ellos". }\end{array}$ & $\begin{array}{l}\text { Es una amenaza constante en las plataformas de } \\
\text { gestión electrónica. Los datos y metadatos son } \\
\text { vulnerables por naturaleza, de ahí la } \\
\text { importancia de la trazabilidad de las acciones } \\
\text { que se realizan en cada uno de los procesos, } \\
\text { auditar todas las acciones que se han realizado } \\
\text { sobre un documento, y la necesidad de aprobar } \\
\text { protocolos de seguridad. } \\
\text { Multiplicar canales de acceso o compartir datos } \\
\text { forma parte de los objetivos y funcionalidades } \\
\text { de las plataformas pero siempre incorporando } \\
\text { las medidas que garanticen las normas de } \\
\text { protección de datos. }\end{array}$ \\
\hline 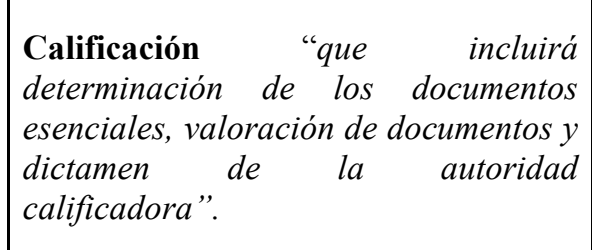 & $\begin{array}{l}\text { Aunque las técnicas, conceptos y metodología } \\
\text { de la selección y evaluación archivística son } \\
\text { trasladables al documento electrónico, lo cierto } \\
\text { es que en nuestro país todavía no se han } \\
\text { aprobado programas de conservación de } \\
\text { documentos electrónicos. }\end{array}$ \\
\hline $\begin{array}{l}\text { Conservación "en función de su valor } \\
\text { y tipo de dictamen de la autoridad } \\
\text { calificadora, a través de la definición } \\
\text { de calendarios de conservación". }\end{array}$ & $\begin{array}{l}\text { La Norma Técnica de Interoperabilidad da por } \\
\text { hecho la existencia, y por tanto la } \\
\text { obligatoriedad, de distintos repositorios en } \\
\text { función de la fase del ciclo de vida en la que se } \\
\text { encuentre el documento electrónico. A nivel }\end{array}$ \\
\hline
\end{tabular}




\begin{tabular}{|c|c|}
\hline $\begin{array}{l}\text { consideraciones para la transferencia } \\
\text { entre repositorios asi como las } \\
\text { responsabilidades en cuanto a su } \\
\text { custodia". }\end{array}$ & $\begin{array}{l}\text { conceptual sigue el modelo OAIS, el sistema de } \\
\text { referencia de archivo electrónico para la } \\
\text { preservación a largo plazo. }\end{array}$ \\
\hline $\begin{array}{l}\text { Eliminación "de los documentos, que } \\
\text { atenderá a la normativa aplicable en } \\
\text { materia de eliminación de Patrimonio } \\
\text { Documental y contemplará la } \\
\text { aplicación de las medidas de seguridad } \\
\text { definidas en el Esquema Nacional de } \\
\text { Seguridad en el ámbito de la } \\
\text { Administración Electrónica". }\end{array}$ & $\begin{array}{l}\text { Una cuestión esencial es separar las } \\
\text { aplicaciones de cliente, los desarrollos en } \\
\text { fuentes no abiertas, de la gestión y acceso al } \\
\text { repositorio de conservación. } \\
\text { Otra cuestión es la caducidad de los certificados } \\
\text { digitales el necesario resellado de los paquetes } \\
\text { de información que forman parte del sistema de } \\
\text { archivo. Unas operaciones de autentificación } \\
\text { que también habrá que realizar sobre los } \\
\text { documentos originalmente en papel que se han } \\
\text { digitalizado en el archivo. Sabiendo, como } \\
\text { ocurre con las capturas realizadas en Registro } \\
\text { General, que el firmado electrónico de esos } \\
\text { ficheros implica automáticamente } \\
\text { imposibilidad de su modificación posterior. } \\
\text { Los documentos en papel que sean } \\
\text { sometidos a procesos de digitalización } \\
\text { certificada tendrán la consideración de copia } \\
\text { auténtica, y se autoriza expresamente su } \\
\text { destrucción. Pensando en los documentos } \\
\text { digitalizados e incorporados al proceso de } \\
\text { tramitación electrónica, en Registro o en las } \\
\text { áreas responsables de gestión, ¿tiene sentido la } \\
\text { conservación del original en papel? El sentido } \\
\text { común aconseja la precaución, al menos en un } \\
\text { primer momento, pero la realidad es otra. Sirva } \\
\text { un ejemplo, en los Sistemas Integrados de } \\
\text { Registro promovidos por la Administración } \\
\text { General del Estado los documentos en papel se } \\
\text { devuelven al ciudadano una vez digitalizados, y } \\
\text { así lo están comenzando a hacer también } \\
\text { algunas administraciones locales. } \\
\text { Si la apuesta es la administración } \\
\text { electrónica, electrónicos tendrán ser todos sus } \\
\text { soportes documentales, evitando prácticas tan } \\
\text { desaconsejables como los sistemas } \\
\text { expedientes híbridos. }\end{array}$ \\
\hline
\end{tabular}

Tabla II. Procesos técnicos en el entorno electrónico. 


\section{CONCLUSIONES.}

Una última reflexión para los que están en la fase de iniciar el camino hacia la eadministración. No hay un mapa del territorio y recorrerlo requiere tiempo, hay que ir paso a paso, aunque inicialmente sean pequeños retos. El único equipaje necesario es el de la actitud. Cada profesional debe elegir el lugar a ocupar a partir de tres elementos que son el soporte de todo proyecto: querer, saber y poder. La motivación se presupone, la capacidad se soluciona aprendiendo, y el poder llevarlo a cabo va a depender de nuestra inteligencia ejecutiva y saber encontrar la estrategia a seguir en función de cada situación. Esa es la habilidad del gestor, pues los nuevos tiempos nos obligan a ser gestores antes que archiveros. En realidad todo se reduce a estar preparado para aprovechar las oportunidades, sabiendo que no se producen por generación espontánea. Si no cambiamos, nada cambia, si hacemos lo que siempre hemos hecho llegaremos siempre al mismo sitio, las oportunidades hay que salir a buscarlas. Es el único modo de ganar el espacio que creemos nos pertenece, somos lo que hacemos, no lo que decimos. Si tu meta es construir el futuro desde el presente, haz el ejercicio de imaginar tu archivo en diez o quince años, traza un plan y actúa, ganar espacio es sólo una cuestión de tiempo.

\section{NOTAS.}

${ }^{1}$ Ley 11/2007, de 22 de junio, de Acceso Electrónico de los Ciudadanos a los Servicios Públicos. Disponible en: <http://www.boe.es/boe/dias/2007/06/23/pdfs/A27150-27166.pdf $>$. [Consulta: 1 de septiembre de 2012].

2 No está de más recordar que han transcurrido casi dos décadas de las X Jornadas de Archivos municipales, donde se abordó monográficamente la problemática derivada de lo que entonces comenzaban a denominarse "nuevos soportes documentales" (Grupo de Archiveros Municipales de Madrid, 1994). También nos puede llamar la atención que la primera edición de TECNIMAP se celebra en Madrid en 1989 y en la segunda convocatoria de Valencia en 1991 ya se abordó el tema de "la validez jurídica del documento informático y las necesarias garantías de privacidad en el manejo de datos personales" Disponible en: $<\mathrm{http}$ :/www.tecnimap.es/es/portal.do?IDM=28\&NM=1>. [Consulta: 1 de septiembre de 2012]. Desde entonces hay que contar por centenares las monografías, artículos, congresos, jornadas o seminarios que han tratado, y seguirán tratando, una cuestión que indiscutiblemente ha alterado por completo el escenario de trabajo en las administraciones locales.

${ }^{3}$ En concreto se indica, en su art. 9 "Transmisión de datos entre Administraciones Públicas", que para un eficaz ejercicio de este derecho "cada Administración deberá facilitar el acceso de las restantes Administraciones Públicas a los datos relativos a los interesados que obren en su poder y se encuentren en soporte electrónico, especificando las condiciones, protocolos y criterios funcionales o técnicos necesarios para acceder a dichos datos con las máximas garantías de seguridad, integridad y disponibilidad, de conformidad con lo dispuesto en la Ley Orgánica 15/1999, de 13 de diciembre, de Protección de Datos de Carácter Personal y su normativa de desarrollo".

${ }^{4}$ La principal novedad de la Ley 11/2007, parcialmente desarrollada por el R.D. 1671/2009, es que ha supuesto el paso de la posibilidad a la obligación por parte de la administración de facilitar la relación con los ciudadanos a través de medios telemáticos. Es su rasgo diferenciador respecto a otras iniciativas legislativas. La Ley 30/1992 de Régimen Jurídico de las Administraciones Públicas y del Procedimiento Administrativo Común supuso un primer impulso ya que recogía en su artículo 45 la posibilidad del empleo de técnicas y medios electrónicos, informáticos y telemáticos, además de la informatización de registros y archivos contemplada en su artículo 38. También la Ley 24/2001 permitía el establecimiento de registros telemáticos para la recepción o salida de solicitudes, escritos y comunicaciones. 
${ }^{5}$ Dado que esa disposición adicional ha sido aplicada muy frecuentemente, en la Ley 2/2011, de 4 de marzo, de Economía Sostenible, se realiza una importante modificación de la Ley 11/2007 en su disposición adicional séptima, especificando que cuando por problemas presupuestarios no se hayan podido implantar los servicios a que hace referencia la Ley 11/2007, "se deberán aprobar y hacer públicos los programas y calendarios de trabajo que sean precisos para ello, atendiendo a las respectivas previsiones presupuestarias, con mención particularizada de las fases en las que los diversos derechos serán exigibles por los ciudadanos", con el importante añadido que "los programas mencionados en el apartado anterior deberán ser objeto de aprobación y publicación en el plazo de seis meses desde la entrada en vigor de la presente Ley", es decir, aparece otra fecha límite para que las entidades con "problemas presupuestarios" aprueben y publiquen estos calendarios de trabajo, incluyendo las fases en las que los diversos derechos puedan ser exigidos por los ciudadanos: 6 de septiembre de 2011. Intuimos que son muy pocos los ayuntamientos que han cumplido este precepto, aunque algunos sí lo han hecho, es el caso de Elche: Plan de Modernización para la administración electrónica (julio 2011-2012). Disponible en $\quad<$ http://www.elche.es/ilive/download/elche/201109/9/Plan\%20de\%20Modernizaci\%C3\%B3n\%20Ayuntamiento\%20de\%20Elche\%20v.1.pdf $>$.

[Consulta: 1 de septiembre de 2012].

${ }^{6}$ La VI Conferencia Europea sobre e-Government se celebró en Poznan (Polonia) el 17 y 18 de noviembre de 2011, bajo el lema Borderless eGovernment Services for Europeans. Disponible en: $<$ http://www.egov2011.pl/egov2011/public/main/>. [Consulta: 1 de septiembre de 2012].

${ }^{7}$ V Conferencia Europea sobre e-Government se celebró en Malmö (Suecia) el 19 y 20 de noviembre de 2009. Disponible en: <http://www.egov2009.se/wp-content/uploads/Ministerial-Declaration-on-eGovernment.pdf $>$. [Consulta: 1 de septiembre de 2012].

${ }^{8}$ En lo que se refiere a los procedimientos de la Administración General del Estado, y según valoraciones del Ministerio de la Presidencia, se estima que el $80 \%$ de los procedimientos administrativos están adaptados a la tramitación electrónica. Merece una mención especial la entrada en funcionamiento del Registro Electrónico Común que posibilita que el ciudadano puede presentar ante la administración local o autonómica cualquier solicitud, escrito o comunicación dirigidas a la AGE y a sus organismos públicos, a través de ORVE, plataforma que interconecta la red de oficinas de registro (presenciales y electrónicas), utilizando la Norma SICRES 3.0 y el Portal de Entidades Locales.

9 United Nations

E-Government Survey 2012. http://unpan1.un.org/intradoc/groups/public/documents/un/unpan048065.pdf>. [Consulta: 1 de septiembre de 2012]. El primer puesto corresponde a Corea del Sur, seguido de Holanda, Reino Unido, Dinamarca y Estados Unidos.

${ }^{10}$ Fundación Orange. Informe anual sobre el desarrollo de la sociedad de la información en España. Año 2011. Madrid: Fundación Orange, 2012. La población de los ayuntamientos que son objeto del estudio oscilan entre los 230.822 habitantes de Elche y los 3.273.049 habitantes de Madrid. Disponible en: $<$ http://www.informeeespana.es/docs/eE2011.pdf>. [Consulta: 1 de septiembre de 2012].

${ }^{11}$ Federación Española de Municipios y Provincias. Grado de adaptación de las Entidades Locales (menores de 20.000 habitantes) a la Ley 11/2007 de acceso electrónico de los ciudadanos a los servicios públicos. Madrid: FEMP, 2011. Disponible en: <http://www.femp.es/files/566-753-archivo/Grado\%20de\%20adaptacion.pdf $>$. [Consulta: 1 de septiembre de 2012].

2 Federación Española de Municipios y Provincias. Experiencias de éxito en e-Administración de las entidades locales. Disponible en: <http://www.femp.es/files/566-1051-archivo/Experiencias\%20de\%20exito\%20en\%20eadministracion\%20en\%20EELL.pdf $>$. [Consulta: 1 de septiembre de 2012].

${ }^{13}$ La Ley 30/2007, de 30 de octubre, de Contratos del Sector Público, que entró en vigor el 1 de Mayo de 2008, establece, en su artículo 42, una importante novedad que pretende asegurar la transparencia y el acceso público a la información a través de Internet, también conocido como perfil de contratante. La sucesión de leyes que han modificado la Ley 30/2007 ha hecho necesaria la aprobación de un texto único: Real Decreto Legislativo 3/2011, de 14 de noviembre, por el que se aprueba el texto refundido de la Ley de Contratos del Sector Público (BOE, $\mathrm{n}^{\circ}$ 276 de 16 de noviembre de 2011). Disponible en: <http://www.boe.es/boe/dias/2011/11/16/pdfs/BOE-A-201117887.pdf $>$. [Consulta: 1 de septiembre de 2012].

14 Referencia citada por M. ROMERO TALLAFIGO en las I Jornadas sobre gestión del patrimonio documental. Córdoba, 29-31 de octubre de 1998. Córdoba: Diputación, 2000. 
15 Sobre el tema del cambio de modelo de archivo son muy interesantes las reflexiones de las directoras de los archivos municipales de Valladolid y Zaragoza: (Feijoo Casado, 2009; Rivás Palá, 2006). En esta misma línea, relatando la experiencia del Ayuntamiento de Leganés en la tramitación electrónica de los procedimientos administrativos (Pérez Almarza, 2010). Igualmente son muy clarificadoras las aportaciones de J. Serra, tomando como referencia experiencias de proyectos de sistemas de gestión documental en entornos electrónicos en la Generalitat de Catalunya (Serra Serra, 2010).

Un buen resumen con estrategias y orientaciones prácticas para implantar un sistema de gestión de documentos electrónicos (Bustelo Ruesta y García Morales, 2008).

16 Propuesta de Agenda digital para España. 25 de julio de 2012. Disponible en: $<$ http://www.agendadigital.gob.es/images/doc/PropuestadeAgendaDigitalparaEspana.pdf $>$. [Consulta: 29 de julio de 2012]. España comparte los objetivos clave de la Agenda Digital para Europa de conseguir que en 2015 más del $50 \%$ de la población utilice la administración electrónica y más del $25 \%$ cumplimenten formularios en línea. Se ha tomado como punto de partida los resultados de diversos diagnósticos como el recientemente publicado por la Comunidad Europea (OECD, 2012).

${ }^{17}$ La entrada en vigor de la Ley de Transparencia, Acceso a la Información Pública y Buen Gobierno será un impulso añadido a los programas de gestión de documentos electrónicos. Se aplicará el principio de publicidad activa a la información institucional, de relevancia jurídica y de naturaleza económica, presupuestaria y estadística. como gastos, adjudicaciones, contratos, o los bienes e ingresos de los cargos públicos. Disponible en: $<$ http://www.leydetransparencia.gob.es/index.htm>. [Consulta: 1 de septiembre de 2012].

18 Cumpliendo con lo establecido en el Real Decreto 1495/2011, de 24 de octubre, por el que se desarrolla la Ley $37 / 2007$, de 16 de noviembre, sobre reutilización de la información del sector público (BOE, $\mathrm{n}^{\circ} 269$, de 8 de noviembre de 2011). Disponible en: < http://www.boe.es/diario_boe/txt.php?id=BOE-A-2011-17560>. [Consulta: 25 de julio de 2012].

19 Resolución de 28 de junio de 2012, de la Secretaría de Estado de Administraciones Públicas, por la que se aprueba la Norma Técnica de Interoperabilidad de Política de gestión de documentos electrónicos (BOE, n 178 , de 26 de julio de 2012). Disponible en: <http://www.boe.es/diario_boe/txt.php?id=BOE-A-2012-10048>. [Consulta: 1 de septiembre de 2012].

${ }^{20}$ La normalización en la gestión de documentos ha cobrado gran importancia en los últimos años. Las ISO 30300 conciben la gestión de los documentos en las organizaciones como un área estratégica (Bustelo Ruesta, 2011) y se complementan con las ISO 15489 (gestión de documentos) ISO 23081 (metadatos para la gestión de documentos), ISO 26122 (análisis de los procesos de trabajo para la gestión de documentos), ISO 13028 (directrices para la implementación de la Digitalización de documentos, ISO 13008 (proceso de migración y conversión de documentos), las ISO 16175 (principios y requisitos funcionales para documentos en entornos electrónicos de oficinas), las ISO 14721 (open archival information system-OAIS), o las ISO 27001 (gestión de Seguridad de la Información). El subcomité internacional ISO TC46/SC11 Archives/Records management, encargado de la elaboración de la serie de normas ISO 30300, ha publicado el whitepaper "Relationship between the ISO 30300 series of standards and other products of ISO/TC 46/SC 11: Records processes and controls", que incluye una aclaratoria tabla que relaciona las normas e informes técnicos sobre gestión de documentos con los procesos de creación y control de documentos del anexo A de la norma ISO 30301: $<$ http://www.iso30300.es/wp-

content/uploads/2012/03/ISOTC46SC11_White_paper_relationship_30300_technical_standards12032012v6.pdf $\rightarrow$. [Consulta: 25 de julio de 2012].

21 Eduardo Nuñez, director del Archivo de Gijón, ha realizado una acertada reflexión sobre este y otros temas relacionados con la aplicación de las Normas ISO en los servicios de archivo (Nuñez Fernández, 2007a y 2007b).

${ }^{22}$ Las funciones del repositorio electrónico están definidas en el capítulo X del R.D. 4/2010, de 8 de enero (Anexo 1), por el que se regula el Esquema Nacional de Interoperabilidad en el ámbito de la Administración Electrónica. La conservación que se puede definir como los "procesos y operaciones que, realizados para garantizar la permanencia intelectual y técnica de documentos auténticos a lo largo del tiempo" (ISO 15489-1 punto 3).

${ }^{23}$ Son muy útiles los trabajos realizados por el CENATIC (Centro Nacional de Referencia de aplicación de las TIC basadas en fuentes abiertas): Disponible en: <http://www.cenatic.es/laecsp/page19/page20/page20.html>.

Anales de Documentación, 2013, vol. 16, nº 1 
[Consulta: 25 de julio de 2012], y el estudio sobre las funcionalidades del archivo electrónico. Disponible en: $<$ http://www.cenatic.es/publicaciones/onsfa?download=30\%3 Asoftware-de-fuentes-abiertas-en-laadministracion-electronica-archivo-electronico-de-las-administraciones-publicas $>$. [Consulta: 25 de julio de 2012]. Hay que mencionar el proyecto iArxiu impulsado por la Generalitat de Catalunya, a través de la Agència Catalana de Certificació (CATCERT), como iniciativa pionera de archivo electrónico orientado a la conservación a largo plazo, aunque por el momento son contados los ayuntamientos catalanes que lo están utilizando y tiene pendiente que su arquitectura de gestión sea en fuentes abiertas. En esta misma línea de proyectos de preservación también hay que mencionar por su proximidad geográfica el modelo desarrollado por la Direcçao Geral de Arquivos de Portugal, con la colaboración técnica de la Universidad do Minho, del Repositório de Objectos Digitais Autênticos (RODA), aunque no deja de ser todavía un modelo conceptual: Disponible en: <http://dgarq.gov.pt/files/2008/10/roda_relatorio1.pdf $>$. [Consulta: 25 de julio de 2012]. Sobre la conservación de documentos electrónicos a largo plazo es de obligada referencia el proyecto InterPARES que tiene como objeto la definición de estrategias para la preservación a largo plazo de la autenticidad de los documentos electrónicos. Al respecto pueden consultarse International Research on Permanent Authentic Records in Electronic Systems (InterPARES) 2: Experiential, Interactive and Dynamic Records. Disponible en: $<$ http://www.interpares.org/ip2/book.cfm>. [Consulta: 1 de septiembre de 2012] y las aportaciones de Alejandro Delgado (Delgado Gómez, 2009).

${ }^{24}$ Este paquete de información encapsulada conforme a una estructura predeterminada, de datos y metadatos, se denomina paquete SIP (Submission Information Package), y formará la unidad de información que ingresará en la plataforma de archivo. El formato más utilizado es recurrir al estándar XML que permite el intercambio de información estructurada entre diferentes plataformas, junto a las especificaciones METS (Metadata Encoding and Transmission Standard).

${ }^{25}$ Estandarizado a través de la ISO 14721:2003 (Consultative Committee for Space Data Systems, 2012). Es necesario mencionar, por su importancia para la preservación, que en el módulo de conservación el paquete SIP se transformado en un paquete AIP (Archival Information Package), que es la estructura en la que la información será almacenada por el repositorio, garantizando la preservación de los contenidos y sus metadatos asociados como de una misma unidad. Cada paquete AIP cuenta con un identificador único y perdurable (PID) que será su referencia en el sistema, el equivalente a la "Signatura" de los archivos convencionales.

${ }^{26}$ Aunque sean frecuentes los problemas de espacio en los servidores, y los sistemas se ralentizan cuando crece el número de ficheros, mucho nos tememos que va a ser excepcional el borrado de documentos electrónicos. La falta de capacidad es más sencillo de solucionar que en los archivos físicos por lo que la tendencia será la de conservar, o en todo caso migrar a otros dispositivos de almacenamiento aunque sea muy lejana la posibilidad de reutilizar esos datos, pero en contadas ocasiones se realizará el borrado de información. Un tipo de práctica que reafirma la necesidad de los sistemas de archivo electrónico de documentos de conservación permanente, y administrados desde el servicio de archivo.

${ }^{27}$ Tal como indica la propia norma, los expedientes electrónicos se componen de documentos electrónicos, índice, firma y metadatos. Con el objeto de favorecer la interoperabilidad y el intercambio de información sería esencial la aprobación de esquemas de metadatos estandarizados tomando como base los metadatos obligatorios que mencionan las Normas. Resolución de 19 de julio de 2011, de la Secretaría de Estado para la Función Pública, por la que se aprueba la Norma Técnica de Interoperabilidad de Expediente Electrónico. Disponible en: < http://www.boe.es/boe/dias/2011/07/30/pdfs/BOE-A-2011-13170.pdf $>$. [Consulta: 30 de septiembre de 2012].

\section{BIBLIOGRAFÍA.}

BUSTELO RUESTA, C. Serie ISO 30300: Sistema de gestión para los documentos [en línea]. Madrid: SEDIC, 2011. Disponible en: <http://www.sedic.es/DT-n2-SEDICISO30300.pdf $>$. [Consulta: 16 de agosto de 2012].

BUSTELO RUESTA, C. y GARCIA-MORALES, E. Guía para la aplicación de la Ley 11 en materia de gestión de documentos electrónicos, expediente electrónico y archivo electrónico [en línea]. Madrid: Ministerio de Cultura, 2008. Disponible en: 
$<$ http://www.archiverosdeandalucia.org/attachments/075_earchivo_en_la_L.11.2007.pdf $>$. [Consulta 1 de septiembre de 2012].

CONSULTATIVE COMMITTEE FOR SPACE DATA SYSTEMS. ISO 14721:2003. Reference Model for an Open Archival Information System (OAIS) [en línea]. Washington: Magenta book, 2012. Disponible en: $<$ http://public.ccsds.org/publications/archive/650x0m2.pdf $>$. [Consultado: $30 \mathrm{de}$ septiembre de 2012].

DELGADO GÓMEZ, A. La conservación a largo plazo de documentos electrónicos: normativa ISO y esfuerzos nacionales e internacionales, Arch-e. Revista Andaluza de Archivos [en línea]. 2009, nº 1. Disponible en:

$<$ http://www.juntadeandalucia.es/cultura/archivos_html/sites/default/contenidos/gener al/revista/numeros/Numero_1/galeria/01-02_Alejandro_Delgado_Gxmez.pdf $>$. [Consultado: 25 de julio de 2012].

FUNDACIÓN ORANGE. Informe anual sobre el desarrollo de la sociedad de la información en España. Año 2011 [en línea]. Madrid: Fundación Orange, 2012. Disponible en: <http://www.informeeespana.es/docs/eE2011.pdf $>$. [Consultado: 1 de septiembre de 2012].

FEDERACIÓN ESPAÑOLA DE MUNICIPIOS Y PROVINCIAS. Grado de adaptación de las Entidades Locales (menores de 20.000 habitantes) a la Ley 11/2007 de acceso electrónico de los ciudadanos a los Servicios Públicos [en línea]. Madrid: FEMP, 2011. Disponible en: <http://www.femp.es/files/566-753archivo/Grado\%20de\%20adaptacion.pdf $>$. [Consultado: 1 de septiembre de 2012].

FEIJOO CASADO, A. La e-administración el paso de la gestión tradicional a la gestión electrónica. Revista General de Información y Documentación [en línea]. 2009, vol. 19, nº 1, p. 161-171. Disponible en: $<$ http://revistas.ucm.es/index.php/RGID/article/view/RGID0909110161A/9147>. [Consultado: 1 de septiembre de 2012].

GRUPO DE ARCHIVEROS MUNICIPALES DE MADRID: Archivos, informática y nuevos soportes documentales. X Jornadas de Archivos Municipales. El Escorial: Comunidad de Madrid, 1994.

NUÑEZ FERNÁNDEZ, E. El camino hacia la normalización y la excelencia: la implantación de normas ISO en los sistemas de gestión de documentos y en los servicios de archivo [en línea]. Lligall, 2007, vol. 26, p. 243-260. Disponible en: $<$ http://www.arxivers.com/publicacions/revista-lligall/edicions-lligall/cat_view/14revista-1ligall/60-1ligall-26.html $>$. [Consultado: 25 de julio de 2012].

OECD e-Government Studies. Reaping the Benefits of ICTs in Spain Strategic Study on Communication Infrastructures and paperless administration [en línea]. Brussels: OECD, 2012. Disponible en: <http://www.oecd.org/gov/50488898.pdf>. [Consultado: 29 de julio de 2012].

PEREZ ALMARZA, V. La e-administración en los ayuntamientos. Problemática real y avances. Revista d'arxius, 2010, $\mathrm{n}^{\circ}$ 9, p. 47-62.

RIVAS PALÁ, E. Nuevos retos para los archivos y los archiveros de la Administración Local. EDOPCA 2006: Administración de documentos y servicios a la ciudadanía en la administración electrónica (I-Europa 2006). [en línea]. Oviedo, 22-24 de 
noviembre de 2006. Disponible en:

$<$ http://ria.asturias.es/RIA/bitstream/123456789/72/1/ElenaRivas_es.pdf $>$.

[Consultado: 1 de septiembre de 2012].

SERRA SERRA, J. La inclusión de requisitos archivísticos en proyectos de tramitación electrónica: el mapa de series y sus formas de implantación. Revista d'arxius, 2010, $\mathrm{n}^{\mathrm{o}}$ 8, p. 295-348. 